\title{
Solvability of a Higher-Order Nonlinear Neutral Delay Difference Equation
}

\author{
Min Liu and Zhenyu Guo \\ School of Sciences, Liaoning Shihua University, Fushun, Liaoning 113001, China \\ Correspondence should be addressed to Zhenyu Guo, guozy@163.com \\ Received 19 March 2010; Revised 10 July 2010; Accepted 5 September 2010 \\ Academic Editor: S. Grace
}

Copyright (C 2010 M. Liu and Z. Guo. This is an open access article distributed under the Creative Commons Attribution License, which permits unrestricted use, distribution, and reproduction in any medium, provided the original work is properly cited.

The existence of bounded nonoscillatory solutions of a higher-order nonlinear neutral delay difference equation $\Delta\left(a_{k n} \cdots \Delta\left(a_{2 n} \Delta\left(a_{1 n} \Delta\left(x_{n}+b_{n} x_{n-d}\right)\right)\right)\right)+f\left(n, x_{n-r_{1 n}}, x_{n-r_{2 n}}, \ldots, x_{n-r_{s n}}\right)=0, n \geq n_{0}$ where $n_{0} \geq 0, d>0, k>0$, and $s>0$ are integers, $\left\{a_{i n}\right\}_{n \geq n_{0}}(i=1,2, \ldots, k)$ and $\left\{b_{n}\right\}_{n \geq n_{0}}$ are real sequences, $\bigcup_{j=1}^{s}\left\{r_{j n}\right\}_{n \geq n_{0}} \subseteq \mathbb{Z}$, and $f:\left\{n: n \geq n_{0}\right\} \times \mathbb{R}^{s} \rightarrow \mathbb{R}$ is a mapping, is studied. Some sufficient conditions for the existence of bounded nonoscillatory solutions of this equation are established by using Schauder fixed point theorem and Krasnoselskii fixed point theorem and expatiated through seven theorems according to the range of value of the sequence $\left\{b_{n}\right\}_{n>n_{0}}$. Moreover, these sufficient conditions guarantee that this equation has not only one bounded nonoscillatory solution but also uncountably many bounded nonoscillatory solutions.

\section{Introduction and Preliminaries}

Recently, the interest in the study of the solvability of difference equations has been increasing (see [1-17] and references cited therein). Some authors have paied their attention to various difference equations. For example,

$$
\Delta\left(a_{n} \Delta x_{n}\right)+p_{n} x_{g(n)}=0, \quad n \geq 0
$$

(see [14]),

$$
\Delta\left(a_{n} \Delta x_{n}\right)=q_{n} x_{n+1}, \quad \Delta\left(a_{n} \Delta x_{n}\right)=q_{n} f\left(x_{n+1}\right), \quad n \geq 0
$$


(see [11]),

$$
\Delta^{2}\left(x_{n}+p x_{n-m}\right)+p_{n} x_{n-k}-q_{n} x_{n-l}=0, \quad n \geq n_{0}
$$

(see [6]),

$$
\Delta^{2}\left(x_{n}+p x_{n-k}\right)+f\left(n, x_{n}\right)=0, \quad n \geq 1
$$

(see [10]),

$$
\Delta^{2}\left(x_{n}-p x_{n-\tau}\right)=\sum_{i=1}^{m} q_{i} f_{i}\left(x_{n-\sigma_{i}}\right), \quad n \geq n_{0}
$$

(see [9]),

$$
\Delta\left(a_{n} \Delta\left(x_{n}+b x_{n-\tau}\right)\right)+f\left(n, x_{n-d_{1 n}}, x_{n-d_{2 n}}, \ldots, x_{n-d_{k n}}\right)=c_{n}, \quad n \geq n_{0}
$$

(see [8]),

$$
\Delta^{m}\left(x_{n}+c x_{n-k}\right)+p_{n} x_{n-r}=0, \quad n \geq n_{0}
$$

(see [15]),

$$
\Delta^{m}\left(x_{n}+c_{n} x_{n-k}\right)+p_{n} f\left(x_{n-r}\right)=0, \quad n \geq n_{0}
$$

(see $[3,4,12,13])$,

$$
\Delta^{m}\left(x_{n}+c x_{n-k}\right)+\sum_{s=1}^{u} p_{n}^{s} f_{s}\left(x_{n-r_{s}}\right)=q_{n}, \quad n \geq n_{0}
$$

(see [16]),

$$
\Delta^{m}\left(x_{n}+c x_{n-k}\right)+p_{n} x_{n-r}-q_{n} x_{n-l}=0, \quad n \geq n_{0}
$$

(see [17]).

Motivated and inspired by the papers mentioned above, in this paper, we investigate the following higher-order nonlinear neutral delay difference equation:

$$
\Delta\left(a_{k n} \cdots \Delta\left(a_{2 n} \Delta\left(a_{1 n} \Delta\left(x_{n}+b_{n} x_{n-d}\right)\right)\right)\right)+f\left(n, x_{n-r_{1 n}}, x_{n-r_{2 n}}, \ldots, x_{n-r_{s n}}\right)=0, \quad n \geq n_{0}
$$

where $n_{0} \geq 0, d>0, k>0$, and $s>0$ are integers, $\left\{a_{i n}\right\}_{n \geq n_{0}}(i=1,2, \ldots, k)$ and $\left\{b_{n}\right\}_{n \geq n_{0}}$ are real sequences, $\bigcup_{j=1}^{s}\left\{r_{j n}\right\}_{n \geq n_{0}} \subseteq \mathbb{Z}$, and $f:\left\{n: n \geq n_{0}\right\} \times \mathbb{R}^{s} \rightarrow \mathbb{R}$ is a mapping. Clearly, difference 
equations (1.1)-(1.10) are special cases of (1.11). By using Schauder fixed point theorem and Krasnoselskii fixed point theorem, the existence of bounded nonoscillatory solutions of (1.11) is established.

Lemma 1.1 (Schauder fixed point theorem). Let $\Omega$ be a nonempty closed convex subset of a Banach space $X$. Let $T: \Omega \rightarrow \Omega$ be a continuous mapping such that $T \Omega$ is a relatively compact subset of $X$. Then $T$ has at least one fixed point in $\Omega$.

Lemma 1.2 (Krasnoselskii fixed point theorem). Let $\Omega$ be a bounded closed convex subset of $a$ Banach space $X$, and let $T_{1}, T_{2}: \Omega \rightarrow X$ satisfy $T_{1} x+T_{2} y \in \Omega$ for each $x, y \in \Omega$. If $T_{1}$ is a contraction mapping and $T_{2}$ is a completely continuous mapping, then the equation $T_{1} x+T_{2} x=x$ has at least one solution in $\Omega$.

The forward difference $\Delta$ is defined as usual, that is, $\Delta x_{n}=x_{n+1}-x_{n}$. The higher-order difference for a positive integer $m$ is defined as $\Delta^{m} x_{n}=\Delta\left(\Delta^{m-1} x_{n}\right), \Delta^{0} x_{n}=x_{n}$. Throughout this paper, assume that $\mathbb{R}=(-\infty,+\infty), \mathbb{N}$ and $\mathbb{Z}$ stand for the sets of all positive integers and integers, respectively, $\alpha=\inf \left\{n-r_{j n}: 1 \leq j \leq s, n \geq n_{0}\right\}, \beta=\min \left\{n_{0}-d, \alpha\right\}, \lim _{n \rightarrow \infty}\left(n-r_{j n}\right)=+\infty$, $1 \leq j \leq s$, and $l_{\beta}^{\infty}$ denotes the set of real sequences defined on the set of positive integers lager than $\beta$ where any individual sequence is bounded with respect to the usual supremum norm $\|x\|=\sup _{n \geq \beta}\left|x_{n}\right|$ for $x=\left\{x_{n}\right\}_{n \geq \beta} \in l_{\beta}^{\infty}$. It is well known that $l_{\beta}^{\infty}$ is a Banach space under the supremum norm. A subset $\Omega$ of a Banach space $X$ is relatively compact if every sequence in $\Omega$ has a subsequence converging to an element of $X$.

Definition 1.3 (see [5]). A set $\Omega$ of sequences in $l_{\beta}^{\infty}$ is uniformly Cauchy (or equi-Cauchy) if, for every $\varepsilon>0$, there exists an integer $N_{0}$ such that

$$
\left|x_{i}-x_{j}\right|<\varepsilon,
$$

whenever $i, j>N_{0}$ for any $x=\left\{x_{k}\right\}_{k \geq \beta}$ in $\Omega$.

Lemma 1.4 (discrete Arzela-Ascoli's theorem [5]). A bounded, uniformly Cauchy subset $\Omega$ of $l_{\beta}^{\infty}$ is relatively compact.

Let

$$
A(M, N)=\left\{x=\left\{x_{n}\right\}_{n \geq \beta} \in l_{\beta}^{\infty}: M \leq x_{n} \leq N, \forall n \geq \beta\right\} \text { for } N>M>0 .
$$

Obviously, $A(M, N)$ is a bounded closed and convex subset of $l_{\beta}^{\infty}$. Put

$$
\bar{b}=\limsup _{n \rightarrow \infty} b_{n}, \quad \underline{b}=\liminf _{n \rightarrow \infty} b_{n} .
$$

By a solution of (1.11), we mean a sequence $\left\{x_{n}\right\}_{n \geq \beta}$ with a positive integer $N_{0} \geq$ $n_{0}+d+|\alpha|$ such that (1.11) is satisfied for all $n \geq N_{0}$. As is customary, a solution of (1.11) is said to be oscillatory about zero, or simply oscillatory, if the terms $x_{n}$ of the sequence $\left\{x_{n}\right\}_{n \geq \beta}$ are neither eventually all positive nor eventually all negative. Otherwise, the solution is called nonoscillatory. 


\section{Existence of Nonoscillatory Solutions}

In this section, a few sufficient conditions of the existence of bounded nonoscillatory solutions of (1.11) are given.

Theorem 2.1. Assume that there exist constants $M$ and $N$ with $N>M>0$ and sequences $\left\{a_{i n}\right\}_{n \geq n_{0}}(1 \leq i \leq k),\left\{b_{n}\right\}_{n \geq n_{0}},\left\{h_{n}\right\}_{n \geq n_{0}}$ and $\left\{q_{n}\right\}_{n \geq n_{0}}$ such that, for $n \geq n_{0}$,

$$
\begin{gathered}
b_{n} \equiv-1, \quad \text { eventually, } \\
\left|f\left(n, u_{1}, u_{2}, \ldots, u_{s}\right)-f\left(n, v_{1}, v_{2}, \ldots, v_{s}\right)\right| \leq h_{n} \max \left\{\left|u_{i}-v_{i}\right|: u_{i}, v_{i} \in[M, N], 1 \leq i \leq s\right\} \\
\left|f\left(n, u_{1}, u_{2}, \ldots, u_{s}\right)\right| \leq q_{n}, \quad u_{i} \in[M, N], 1 \leq i \leq s \\
\sum_{t=n_{0}}^{\infty} \max \left\{\frac{1}{\left|a_{i t}\right|}, h_{t}, q_{t}: 1 \leq i \leq k\right\}<+\infty
\end{gathered}
$$

Then (1.11) has a bounded nonoscillatory solution in $A(M, N)$.

Proof. Choose $L \in(M, N)$. By (2.1), (2.4), and the definition of convergence of series, an integer $N_{0}>n_{0}+d+|\alpha|$ can be chosen such that

$$
\begin{gathered}
b_{n} \equiv-1, \quad \forall n \geq N_{0} \\
\sum_{j=1}^{\infty} \sum_{t_{1}=N_{0}+j d}^{\infty} \sum_{t_{2}=t_{1}}^{\infty} \cdots \sum_{t_{k}=t_{k-1}}^{\infty} \sum_{t=t_{k}}^{\infty} \frac{q_{t}}{\left|\prod_{i=1}^{k} a_{i t_{i}}\right|} \leq \min \{L-M, N-L\} .
\end{gathered}
$$

Define a mapping $T_{L}: A(M, N) \rightarrow X$ by

$$
\left(T_{L} x\right)_{n}= \begin{cases}L-(-1)^{k} \sum_{j=1}^{\infty} \sum_{t_{1}=n+j d}^{\infty} \sum_{t_{2}=t_{1}}^{\infty} \cdots \sum_{t_{k}=t_{k-1}}^{\infty} \sum_{t=t_{k}}^{\infty} \frac{f\left(t, x_{t-r_{1 t}}, x_{t-r_{2 t}}, \ldots, x_{t-r_{s t}}\right)}{\prod_{i=1}^{k} a_{i t_{i}}}, & n \geq N_{0}, \\ \left(T_{L} x\right)_{N_{0}}, & \beta \leq n<N_{0}\end{cases}
$$

for all $x \in A(M, N)$.

(i) It is claimed that $T_{L} x \in A(M, N)$, for all $x \in A(M, N)$. 
In fact, for every $x \in A(M, N)$ and $n \geq N_{0}$, it follows from (2.3) and (2.6) that

$$
\begin{aligned}
\left(T_{L} x\right)_{n} & \geq L-\sum_{j=1}^{\infty} \sum_{t_{1}=n+j d}^{\infty} \sum_{t_{2}=t_{1}}^{\infty} \cdots \sum_{t_{k}=t_{k-1}}^{\infty} \sum_{t=t_{k}}^{\infty} \frac{\left|f\left(t, x_{t-r_{1 t}}, x_{t-r_{2 t}}, \ldots, x_{t-r_{s t}}\right)\right|}{\left|\prod_{i=1}^{k} a_{i t_{i}}\right|} \\
& \geq L-\sum_{j=1}^{\infty} \sum_{t_{1}=N_{0}+j d}^{\infty} \sum_{t_{2}=t_{1}}^{\infty} \cdots \sum_{t_{k}=t_{k-1}}^{\infty} \sum_{t=t_{k}}^{\infty} \frac{q_{t}}{\left|\prod_{i=1}^{k} a_{i t_{i}}\right|} \\
& \geq M, \\
\left(T_{L} x\right)_{n} & \leq L+\sum_{j=1}^{\infty} \sum_{t_{1}=N_{0}+j d}^{\infty} \sum_{t_{2}=t_{1}}^{\infty} \ldots \sum_{t_{k}=t_{k-1}}^{\infty} \sum_{t=t_{k}}^{\infty} \frac{q_{t}}{\left|\prod_{i=1}^{k} a_{i t_{i}}\right|} \\
& \leq N .
\end{aligned}
$$

That is, $\left(T_{L} x\right)(A(M, N)) \subseteq A(M, N)$.

(ii) It is declared that $T_{L}$ is continuous.

Let $x=\left\{x_{n}\right\} \in A(M, N)$ and $x^{(u)}=\left\{x_{n}^{(u)}\right\} \in A(M, N)$ be any sequence such that $x_{n}^{(u)} \rightarrow x_{n}$ as $u \rightarrow \infty$. For $n \geq N_{0}$, (2.2) guarantees that

$$
\begin{aligned}
& \left|T_{L} x_{n}^{(u)}-T_{L} x_{n}\right| \\
& \quad \leq \sum_{j=1}^{\infty} \sum_{t_{1}=n+j d}^{\infty} \sum_{t_{2}=t_{1}}^{\infty} \cdots \sum_{t_{k}=t_{k-1}}^{\infty} \sum_{t=t_{k}}^{\infty} \frac{\left|f\left(t, x_{t-r_{1 t}}^{(u)}, x_{t-r_{2 t}}^{(u)}, \ldots, x_{t-r_{s t}}^{(u)}\right)-f\left(t, x_{t-r_{1 t}}, x_{t-r_{2 t}}, \ldots, x_{t-r_{s t}}\right)\right|}{\left|\prod_{i=1}^{k} a_{i t_{i}}\right|} \\
& \quad \leq \sum_{j=1}^{\infty} \sum_{t_{1}=n+j d}^{\infty} \sum_{t_{2}=t_{1}}^{\infty} \cdots \sum_{t_{k}=t_{k-1}}^{\infty} \sum_{t=t_{k}}^{\infty} \frac{h_{t} \max \left\{\left|x_{t-r_{j t}}^{(u)}-x_{t-r_{j t} \mid}\right|: 1 \leq j \leq s\right\}}{\left|\prod_{i=1}^{k} a_{i t_{i}}\right|} \\
& \quad \leq\left\|x^{(u)}-x\right\| \sum_{j=1}^{\infty} \sum_{t_{1}=N_{0}+j d}^{\infty} \sum_{t_{2}=t_{1}}^{\infty} \cdots \sum_{t_{k}=t_{k-1}}^{\infty} \sum_{t=t_{k}}^{\infty} \frac{h_{t}}{\left|\prod_{i=1}^{k} a_{i t_{i}}\right|} .
\end{aligned}
$$

This inequality and (2.4) imply that $T_{L}$ is continuous.

(iii) It can be asserted that $T_{L} A(M, N)$ is relatively compact. 
By (2.4), for any $\varepsilon>0$, take $N_{3} \geq N_{0}$ large enough so that

$$
\sum_{j=1}^{\infty} \sum_{t_{1}=N_{3}+j d}^{\infty} \sum_{t_{2}=t_{1}}^{\infty} \cdots \sum_{t_{k}=t_{k-1}}^{\infty} \sum_{t=t_{k}}^{\infty} \frac{q_{t}}{\left|\prod_{i=1}^{k} a_{i t_{i}}\right|}<\frac{\varepsilon}{2} .
$$

Then, for any $x=\left\{x_{n}\right\} \in A(M, N)$ and $n_{1}, n_{2} \geq N_{3}$, (2.10) ensures that

$$
\begin{aligned}
\left|T_{L} x_{n_{1}}-T_{L} x_{n_{2}}\right| \leq & \sum_{j=1}^{\infty} \sum_{t_{1}=n_{1}+j d}^{\infty} \sum_{t_{2}=t_{1}}^{\infty} \cdots \sum_{t_{k}=t_{k-1}}^{\infty} \sum_{t=t_{k}}^{\infty} \frac{\left|f\left(t, x_{t-r_{1 t}}, x_{t-r_{2 t}}, \ldots, x_{t-r_{s t}}\right)\right|}{\left|\prod_{i=1}^{k} a_{i t_{i}}\right|} \\
& +\sum_{j=1}^{\infty} \sum_{t_{1}=n_{2}+j d}^{\infty} \sum_{t_{2}=t_{1}}^{\infty} \cdots \sum_{t_{k}=t_{k-1}}^{\infty} \sum_{t=t_{k}}^{\infty} \frac{\left|f\left(t, x_{t-r_{1},}, x_{t-r_{2 t}}, \ldots, x_{t-r_{s t}}\right)\right|}{\left|\prod_{i=1}^{k} a_{i t_{i}}\right|} \\
\leq & \sum_{j=1}^{\infty} \sum_{t_{1}=N_{3}+j d}^{\infty} \sum_{t_{2}=t_{1}}^{\infty} \cdots \sum_{t_{k}=t_{k-1}}^{\infty} \sum_{t=t_{k}}^{\infty} \frac{q_{t}}{\left|\prod_{i=1}^{k} a_{i t_{i}}\right|} \\
& +\sum_{j=1}^{\infty} \sum_{t_{1}=N_{3}+j d}^{\infty} \sum_{t_{2}=t_{1}}^{\infty} \cdots \sum_{t_{k}=t_{k-1}}^{\infty} \sum_{t=t_{k}}^{\infty} \frac{q_{t}}{\left|\prod_{i=1}^{k} a_{i t_{i}}\right|} \\
< & \frac{\varepsilon}{2}+\frac{\varepsilon}{2}=\varepsilon
\end{aligned}
$$

which means that $T_{L} A(M, N)$ is uniformly Cauchy. Therefore, by Lemma 1.4, $T_{L} A(M, N)$ is relatively compact.

By Lemma 1.1, there exists $x=\left\{x_{n}\right\} \in A(M, N)$ such that $T_{L} x=x$, which is a bounded nonoscillatory solution of (1.11). In fact, for $n \geq N_{0}+d$,

$$
\begin{gathered}
x_{n}=L-(-1)^{k} \sum_{j=1}^{\infty} \sum_{t_{1}=n+j d}^{\infty} \sum_{t_{2}=t_{1}}^{\infty} \cdots \sum_{t_{k}=t_{k-1}}^{\infty} \sum_{t=t_{k}}^{\infty} \frac{f\left(t, x_{t-r_{1 t}}, x_{t-r_{2 t}}, \ldots, x_{t-r_{s t}}\right)}{\prod_{i=1}^{k} a_{i t_{i}}}, \\
x_{n-d}=L-(-1)^{k} \sum_{j=1}^{\infty} \sum_{t_{1}=n+(j-1)}^{\infty} \sum_{d}^{\infty} \cdots \sum_{t_{2}=t_{1}}^{\infty} \sum_{t_{k}=t_{k-1}}^{\infty} \frac{f\left(t, x_{t-r_{1 t}}, x_{t-r_{2 t}}, \ldots, x_{t-r_{s t}}\right)}{\prod_{i=1}^{k} a_{i t_{i}}},
\end{gathered}
$$


which derives that

$$
\begin{aligned}
x_{n}-x_{n-d}= & (-1)^{k} \sum_{j=1}^{\infty} \sum_{t_{1}=n+(j-1)}^{n+j d-1} \sum_{t_{2}=t_{1}}^{\infty} \cdots \sum_{t_{k}=t_{k-1}}^{\infty} \sum_{t=t_{k}}^{\infty} \frac{f\left(t, x_{t-r_{1 t}}, x_{t-r_{2 t}}, \ldots, x_{t-r_{s t}}\right)}{\prod_{i=1}^{k} a_{i t_{i}}} \\
\Delta\left(x_{n}-x_{n-d}\right)= & (-1)^{k} \sum_{j=1}^{\infty} \sum_{t_{1}=n+1+(j-1)}^{n+j d} \sum_{t_{2}=t_{1}}^{\infty} \cdots \sum_{t_{k}=t_{k-1}}^{\infty} \sum_{t=t_{k}}^{\infty} \frac{f\left(t, x_{t-r_{1 t}}, x_{t-r_{2 t}}, \ldots, x_{t-r_{s t}}\right)}{\prod_{i=1}^{k} a_{i t_{i}}} \\
& -(-1)^{k} \sum_{j=1}^{\infty} \sum_{t_{1}=n+(j-1) d}^{n+j d-1} \sum_{t_{2}=t_{1}}^{\infty} \cdots \sum_{t_{k}=t_{k-1}}^{\infty} \sum_{t=t_{k}}^{\infty} \frac{f\left(t, x_{t-r_{1 t}}, x_{t-r_{2 t}}, \ldots, x_{t-r_{s t}}\right)}{\prod_{i=1}^{k} a_{i t_{i}}} \\
= & -(-1)^{k} \sum_{j=1}^{\infty} \sum_{t_{2}=n+(j-1) d}^{\infty} \sum_{t_{3}=t_{2}}^{\infty} \cdots \sum_{t_{k}=t_{k-1}}^{\infty} \sum_{t=t_{k}}^{\infty} \frac{f\left(t, x_{t-r_{1 t}}, x_{t-r_{2 t}}, \ldots, x_{t-r_{s t}}\right)}{a_{1(n+(j-1) d)} \prod_{i=2}^{k} a_{i t_{i}}} \\
& +(-1)^{k} \sum_{j=1}^{\infty} \sum_{t_{2}=n+j d}^{\infty} \sum_{t_{3}=t_{2}}^{\infty} \ldots \sum_{t_{k}=t_{k-1}}^{\infty} \sum_{t=t_{k}}^{\infty} \frac{f\left(t, x_{t-r_{1 t}}, x_{t-r_{2 t}}, \ldots, x_{t-r_{s t}}\right)}{a_{1(n+j d)} \prod_{i=2}^{k} a_{i t_{i}}} \\
= & (-1)^{k-1} \sum_{t_{2}=n}^{\infty} \sum_{t_{3}=t_{2}}^{\infty} \ldots \sum_{t_{k}=t_{k-1}}^{\infty} \sum_{t=t_{k}}^{\infty} \frac{f\left(t, x_{t-r_{1 t}}, x_{t-r_{2 t}}, \ldots, x_{t-r_{s t}}\right)}{a_{1 n} \prod_{i=2}^{k} a_{i t_{i}}}
\end{aligned}
$$

That is,

$$
a_{1 n} \Delta\left(x_{n}-x_{n-d}\right)=(-1)^{k-1} \sum_{t_{2}=n}^{\infty} \sum_{t_{3}=t_{2}}^{\infty} \cdots \sum_{t_{k}=t_{k-1}}^{\infty} \sum_{t=t_{k}}^{\infty} \frac{f\left(t, x_{t-r_{1 t}}, x_{t-r_{2 t}}, \ldots, x_{t-r_{s t}}\right)}{\prod_{i=2}^{k} a_{i t_{i}}}
$$

by which it follows that

$$
\begin{aligned}
\Delta\left(a_{1 n} \Delta\left(x_{n}-x_{n-d}\right)\right)= & (-1)^{k-1} \sum_{t_{2}=n+1}^{\infty} \sum_{t_{3}=t_{2}}^{\infty} \cdots \sum_{t_{k}=t_{k-1}}^{\infty} \sum_{t=t_{k}}^{\infty} \frac{f\left(t, x_{t-r_{1 t}}, x_{t-r_{2 t}}, \ldots, x_{t-r_{s t}}\right)}{\prod_{i=2}^{k} a_{i t_{i}}} \\
& -(-1)^{k-1} \sum_{t_{2}=n}^{\infty} \sum_{t_{3}=t_{2}}^{\infty} \cdots \sum_{t_{k}=t_{k-1}}^{\infty} \sum_{t=t_{k}}^{\infty} \frac{f\left(t, x_{t-r_{1 t}}, x_{t-r_{2 t}}, \ldots, x_{t-r_{s t}}\right)}{\prod_{i=2}^{k} a_{i t_{i}}} \\
= & (-1)^{k-2} \sum_{t_{3}=n}^{\infty} \sum_{t_{4}=t_{3}}^{\infty} \cdots \sum_{t_{k}=t_{k-1}}^{\infty} \sum_{t=t_{k}}^{\infty} \frac{f\left(t, x_{t-r_{1 t}}, x_{t-r_{2 t}}, \ldots, x_{t-r_{s t}}\right)}{a_{2 n} \prod_{i=3}^{k} a_{i t_{i}}}, \\
\vdots & \\
\Delta\left(a_{k n} \cdots \Delta\left(a_{2 n} \Delta\left(a_{1 n} \Delta\left(x_{n}+b_{n} x_{n-d}\right)\right)\right)\right)= & (-1)^{k-(k+1)} f\left(n, x_{n-r_{1 n}}, x_{n-r_{2 n}}, \ldots, x_{n-r_{s n}}\right) \\
= & -f\left(n, x_{n-r_{1 n}}, x_{n-r_{2 n}}, \ldots, x_{n-r_{s n}}\right) .
\end{aligned}
$$

Therefore, $x$ is a bounded nonoscillatory solution of (1.11). This completes the proof. 
Remark 2.2. The conditions of Theorem 2.1 ensure the (1.11) has not only one bounded nonoscillatory solution but also uncountably many bounded nonoscillatory solutions. In fact, let $L_{1}, L_{2} \in(M, N)$ with $L_{1} \neq L_{2}$. For $L_{1}$ and $L_{2}$, as the preceding proof in Theorem 2.1, there exist integers $N_{1}, N_{2} \geq n_{0}+d+|\alpha|$ and mappings $T_{L_{1}}, T_{L_{2}}$ satisfying (2.5)-(2.7), where $L, N_{0}$ are replaced by $L_{1}, N_{1}$ and $L_{2}, N_{2}$, respectively, and $\sum_{j=1}^{\infty} \sum_{t_{1}=N_{4}+j d}^{\infty} \sum_{t_{2}=t_{1}}^{\infty} \cdots \sum_{t_{k}=t_{k-1}}^{\infty} \sum_{t=t_{k}}^{\infty}\left(h_{t} /\left|\prod_{i=1}^{k} a_{i t_{i}}\right|\right)<\left|L_{1}-L_{2}\right| / 2 N$ for some $N_{4} \geq$ $\max \left\{N_{1}, N_{2}\right\}$. Then the mappings $T_{L_{1}}$ and $T_{L_{2}}$ have fixed points $x, y \in A(M, N)$, respectively, which are bounded nonoscillatory solutions of (1.11) in $A(M, N)$. For the sake of proving that (1.11) possesses uncountably many bounded nonoscillatory solutions in $A(M, N)$, it is only needed to show that $x \neq y$. In fact, by (2.7), we know that, for $n \geq N_{4}$,

$$
\begin{aligned}
& x_{n}=L_{1}-(-1)^{k} \sum_{j=1}^{\infty} \sum_{t_{1}=n+j}^{\infty} \sum_{t_{2}=t_{1}}^{\infty} \cdots \sum_{t_{k}=t_{k-1}}^{\infty} \sum_{t=t_{k}}^{\infty} \frac{f\left(t, x_{t-r_{1 t}}, x_{t-r_{2 t}}, \ldots, x_{t-r_{s t}}\right)}{\prod_{i=1}^{k} a_{i t_{i}}}, \\
& y_{n}=L_{2}-(-1)^{k} \sum_{j=1}^{\infty} \sum_{t_{1}=n+j}^{\infty} \sum_{d=t_{2}=t_{1}}^{\infty} \ldots \sum_{t_{k}=t_{k-1}}^{\infty} \sum_{t=t_{k}}^{\infty} \frac{f\left(t, y_{t-r_{1 t}}, y_{t-r_{2 t}}, \ldots, y_{t-r_{s t}}\right)}{\prod_{i=1}^{k} a_{i t_{i}}} .
\end{aligned}
$$

Then,

$$
\begin{aligned}
\left|x_{n}-y_{n}\right| \geq & \left|L_{1}-L_{2}\right| \\
& -\sum_{j=1}^{\infty} \sum_{t_{1}=n+j d}^{\infty} \sum_{t_{2}=t_{1}}^{\infty} \cdots \sum_{t_{k}=t_{k-1}}^{\infty} \sum_{t=t_{k}}^{\infty} \frac{\left|f\left(t, x_{t-r_{1 t}}, x_{t-r_{2 t}}, \ldots, x_{t-r_{s t}}\right)-f\left(t, y_{t-r_{1 t}}, y_{t-r_{2 t}}, \ldots, y_{t-r_{s t}}\right)\right|}{\left|\prod_{i=1}^{k} a_{i t_{i}}\right|} \\
\geq & \left|L_{1}-L_{2}\right|-\|x-y\| \sum_{j=1}^{\infty} \sum_{t_{1}=N_{4}+j d}^{\infty} \sum_{t_{2}=t_{1}}^{\infty} \cdots \sum_{t_{k}=t_{k-1}}^{\infty} \sum_{t=t_{k}}^{\infty} \frac{h_{t}}{\left|\prod_{i=1}^{k} a_{i t_{i}}\right|} \\
\geq & \left|L_{1}-L_{2}\right|-2 N \sum_{j=1}^{\infty} \sum_{t_{1}=N_{4}+j d}^{\infty} \sum_{t_{2}=t_{1}}^{\infty} \cdots \sum_{t_{k}=t_{k-1}}^{\infty} \sum_{t=t_{k}}^{\infty} \frac{h_{t}}{\left|\prod_{i=1}^{k} a_{i t_{i}}\right|} \\
> & 0, \quad n \geq N_{4},
\end{aligned}
$$

that is, $x \neq y$.

Theorem 2.3. Assume that there exist constants $M$ and $N$ with $N>M>0$ and sequences $\left\{a_{i n}\right\}_{n \geq n_{0}}(1 \leq i \leq k),\left\{b_{n}\right\}_{n \geq n_{0}},\left\{h_{n}\right\}_{n \geq n_{0}},\left\{q_{n}\right\}_{n \geq n_{0}}$ satisfying (2.2)-(2.4) and

$$
b_{n} \equiv 1, \quad \text { eventually }
$$

Then (1.11) has a bounded nonoscillatory solution in $A(M, N)$. 
Proof. Choose $L \in(M, N)$. By (2.18) and (2.4), an integer $N_{0}>n_{0}+d+|\alpha|$ can be chosen such that

$$
\begin{gathered}
b_{n} \equiv 1, \quad \forall n \geq N_{0} \\
\sum_{j=1}^{\infty} \sum_{t_{1}=N_{0}+(2 j-1) d}^{N_{0}+2 j d-1} \sum_{t_{2}=t_{1}}^{\infty} \cdots \sum_{t_{k}=t_{k-1}}^{\infty} \sum_{t=t_{k}}^{\infty} \frac{q_{t}}{\left|\prod_{i=1}^{k} a_{i t_{i}}\right|} \leq \min \{L-M, N-L\}
\end{gathered}
$$

Define a mapping $T_{L}: A(M, N) \rightarrow X$ by

$$
\left(T_{L} x\right)_{n}=\left\{\begin{array}{cl}
L+(-1)^{k} \sum_{j=1}^{\infty} \sum_{t_{1}=n+(2 j-1) d}^{n+2 j d-1} \sum_{t_{2}=t_{1}}^{\infty} & \\
\cdots \sum_{t_{k}=t_{k-1}}^{\infty} \sum_{t=t_{k}}^{\infty} \frac{f\left(t, x_{t-r_{1 t}}, x_{t-r_{2 t}}, \ldots, x_{t-r_{s t}}\right)}{\prod_{i=1}^{k} a_{i t_{i}}}, & n \geq N_{0}, \\
\left(T_{L} x\right)_{N_{0}}, & \beta \leq n<N_{0}
\end{array}\right.
$$

for all $x \in A(M, N)$.

The proof that $T_{L}$ has a fixed point $x=\left\{x_{n}\right\} \in A(M, N)$ is analogous to that in Theorem 2.1. It is claimed that the fixed point $x$ is a bounded nonoscillatory solution of (1.11). In fact, for $n \geq N_{0}+d$,

$$
\begin{gathered}
x_{n}=L+(-1)^{k} \sum_{j=1}^{\infty} \sum_{t_{1}=n+(2 j-1) d}^{n+2 j d-1} \sum_{t_{2}=t_{1}}^{\infty} \cdots \sum_{t_{k}=t_{k-1}}^{\infty} \sum_{t=t_{k}}^{\infty} \frac{f\left(t, x_{t-r_{1 t}}, x_{t-r_{2 t}}, \ldots, x_{t-r_{s t}}\right)}{\prod_{i=1}^{k} a_{i t_{i}}}, \\
x_{n-d}=L+(-1)^{k} \sum_{j=1}^{\infty} \sum_{t_{1}=n+2(j-1) d}^{n+(2 j-1) d-1} \sum_{t_{2}=t_{1}}^{\infty} \cdots \sum_{t_{k}=t_{k-1}}^{\infty} \sum_{t=t_{k}}^{\infty} \frac{f\left(t, x_{t-r_{1 t}}, x_{t-r_{2 t}}, \ldots, x_{t-r_{s t}}\right)}{\prod_{i=1}^{k} a_{i t_{i}}},
\end{gathered}
$$

by which it follows that

$$
x_{n}+x_{n-d}=2 L+(-1)^{k} \sum_{j=1}^{\infty} \sum_{t_{1}=n+(j-1)}^{n+j d-1} \sum_{t_{2}=t_{1}}^{\infty} \cdots \sum_{t_{k}=t_{k-1}}^{\infty} \sum_{t=t_{k}}^{\infty} \frac{f\left(t, x_{t-r_{1 t}}, x_{t-r_{2 t}} \ldots, x_{t-r_{s t}}\right)}{\prod_{i=1}^{k} a_{i t_{i}}} .
$$

The rest of the proof is similar to that in Theorem 2.1. This completes the proof.

Theorem 2.4. Assume that there exist constants $b, M$, and $N$ with $N>M>0$ and sequences $\left\{a_{i n}\right\}_{n \geq n_{0}}(1 \leq i \leq k),\left\{b_{n}\right\}_{n \geq n_{0}},\left\{h_{n}\right\}_{n \geq n_{0}},\left\{q_{n}\right\}_{n \geq n_{0}}$, satisfying (2.2)-(2.4) and

$$
\left|b_{n}\right| \leq b<\frac{N-M}{2 N}, \quad \text { eventually. }
$$

Then (1.11) has a bounded nonoscillatory solution in $A(M, N)$. 
Proof. Choose $L \in(M+b N, N-b N)$. By (2.23) and (2.4), an integer $N_{0}>n_{0}+d+|\alpha|$ can be chosen such that

$$
\begin{gathered}
\left|b_{n}\right| \leq b<\frac{N-M}{2 N}, \quad \forall n \geq N_{0}, \\
\sum_{t_{1}=N_{0}}^{\infty} \sum_{t_{2}=t_{1}}^{\infty} \cdots \sum_{t_{k}=t_{k-1}}^{\infty} \sum_{t=t_{k}}^{\infty} \frac{q_{t}}{\left|\prod_{i=1}^{k} a_{i t_{i}}\right|} \leq \min \{L-b N-M, N-b N-L\} .
\end{gathered}
$$

Define two mappings $T_{1 L}, T_{2 L}: A(M, N) \rightarrow X$ by

$$
\begin{aligned}
& \left(T_{1 L} x\right)_{n}= \begin{cases}L-b_{n} x_{n-d}, & n \geq N_{0}, \\
\left(T_{1 L} x\right)_{N_{0}}, & \beta \leq n<N_{0},\end{cases} \\
& \left(T_{2 L} x\right)_{n}= \begin{cases}(-1)^{k} \sum_{t_{1}=n}^{\infty} \sum_{t_{2}=t_{1}}^{\infty} \cdots \sum_{t_{k}=t_{k-1}}^{\infty} \sum_{t=t_{k}}^{\infty} \frac{f\left(t, x_{t-r_{1 t}}, x_{t-r_{2 t}}, \ldots, x_{t-r_{s t}}\right)}{\prod_{i=1}^{k} a_{i t_{i}}}, & n \geq N_{0}, \\
\left(T_{2 L} x\right)_{N_{0}}, & \beta \leq n<N_{0}\end{cases}
\end{aligned}
$$

for all $x \in A(M, N)$.

(i) It is claimed that $T_{1 L} x+T_{2 L} y \in A(M, N)$, for all $x, y \in A(M, N)$.

In fact, for every $x, y \in A(M, N)$ and $n \geq N_{0}$, it follows from (2.3), (2.24) that

$$
\begin{aligned}
& \left(T_{1 L} x+T_{2 L} y\right)_{n} \geq L-b N-\sum_{t_{1}=N_{0}}^{\infty} \sum_{t_{2}=t_{1}}^{\infty} \cdots \sum_{t_{k}=t_{k-1}}^{\infty} \sum_{t=t_{k}}^{\infty} \frac{q_{t}}{\left|\prod_{i=1}^{k} a_{i t_{i}}\right|} \geq M, \\
& \left(T_{1 L} x+T_{2 L} y\right)_{n} \leq L+b N+\sum_{t_{1}=N_{0}}^{\infty} \sum_{t_{2}=t_{1}}^{\infty} \cdots \sum_{t_{k}=t_{k-1}}^{\infty} \sum_{t=t_{k}}^{\infty} \frac{q_{t}}{\left|\prod_{i=1}^{k} a_{i t_{i}}\right|} \leq N
\end{aligned}
$$

That is, $\left(T_{1 L} x+T_{2 L} y\right)(A(M, N)) \subseteq A(M, N)$.

(ii) It is declared that $T_{1 L}$ is a contraction mapping on $A(M, N)$.

In reality, for any $x, y \in A(M, N)$ and $n \geq N_{0}$, it is easy to derive that

$$
\left|\left(T_{1 L} x\right)_{n}-\left(T_{1 L} y\right)_{n}\right| \leq\left|b_{n}\right|\left|x_{n-d}-y_{n-d}\right| \leq b\|x-y\|
$$

which implies that

$$
\left\|T_{1 L} x-T_{1 L} y\right\| \leq b\|x-y\| .
$$

Then, $b<(N-M) / 2 N<1$ ensures that $T_{1 L}$ is a contraction mapping on $A(M, N)$.

(iii) Similar to (ii) and (iii) in the proof of Theorem 2.1, it can be showed that $T_{2 L}$ is completely continuous.

By Lemma 1.2, there exists $x=\left\{x_{n}\right\} \in A(M, N)$ such that $T_{1 L} x+T_{2 L} x=x$, which is a bounded nonoscillatory solution of (1.11). This completes the proof. 
Theorem 2.5. Assume that there exist constants $M$ and $N$ with $N>((2-\underline{b}) /(1-\bar{b})) M>0$ and sequences $\left\{a_{i n}\right\}_{n \geq n_{0}}(1 \leq i \leq k),\left\{b_{n}\right\}_{n \geq n_{0}},\left\{h_{n}\right\}_{n \geq n_{0}},\left\{q_{n}\right\}_{n \geq n_{0}}$ satisfying (2.2)-(2.4) and

$$
b_{n} \geq 0 \text {, eventually, and } 0 \leq \underline{b} \leq \bar{b}<1 \text {. }
$$

Then (1.11) has a bounded nonoscillatory solution in $A(M, N)$.

Proof. Choose $L \in(M+((1+\bar{b}) / 2) N, N+(\underline{b} / 2) M)$. By (2.29) and (2.4), an integer $N_{0}>$ $n_{0}+d+|\alpha|$ can be chosen such that

$$
\begin{gathered}
\frac{\underline{b}}{2} \leq b_{n} \leq \frac{1+\bar{b}}{2}, \quad \forall n \geq N_{0} \\
\sum_{t_{1}=N_{0}}^{\infty} \sum_{t_{2}=t_{1}}^{\infty} \cdots \sum_{t_{k}=t_{k-1}}^{\infty} \sum_{t=t_{k}}^{\infty} \frac{q_{t}}{\left|\prod_{i=1}^{k} a_{i t_{i}}\right|} \leq \min \left\{L-M-\frac{1+\bar{b}}{2} N, N-L+\frac{b}{2} M\right\} .
\end{gathered}
$$

Define two mappings $T_{1 L}, T_{2 L}: A(M, N) \rightarrow X$ as (2.25). The rest of the proof is analogous to that in Theorem 2.4. This completes the proof.

Similar to the proof of Theorem 2.5, we have the following theorem.

Theorem 2.6. Assume that there exist constants $M$ and $N$ with $N>((2+\bar{b}) /(1+\underline{b})) M>0$ and sequences $\left\{a_{i n}\right\}_{n \geq n_{0}}(1 \leq i \leq k),\left\{b_{n}\right\}_{n \geq n_{0}},\left\{h_{n}\right\}_{n \geq n_{0}},\left\{q_{n}\right\}_{n \geq n_{0}}$ satisfying (2.2)-(2.4) and

$$
b_{n} \leq 0 \text {, eventually, and }-1<\underline{b} \leq \bar{b} \leq 0 .
$$

Then (1.11) has a bounded nonoscillatory solution in $A(M, N)$.

Theorem 2.7. Assume that there exist constants $M$ and $N$ with $N>\left(\underline{b}\left(\bar{b}^{2}-\underline{b}\right) / \bar{b}\left(\underline{b}^{2}-\bar{b}\right)\right) M>0$ and sequences $\left\{a_{i n}\right\}_{n \geq n_{0}}(1 \leq i \leq k),\left\{b_{n}\right\}_{n \geq n_{0^{\prime}}}\left\{h_{n}\right\}_{n \geq n_{0}},\left\{q_{n}\right\}_{n \geq n_{0}}$ satisfying (2.2)-(2.4) and

$$
b_{n}>1 \text {, eventually, } 1<\underline{b} \text { and } \bar{b}<\underline{b}^{2}<+\infty \text {. }
$$

Then (1.11) has a bounded nonoscillatory solution in $A(M, N)$.

Proof. Take $\varepsilon \in(0, \underline{b}-1)$ sufficiently small satisfying

$$
\begin{gathered}
1<\underline{b}-\varepsilon<\bar{b}+\varepsilon<(\underline{b}-\varepsilon)^{2} \\
\left((\bar{b}+\varepsilon)(\underline{b}-\varepsilon)^{2}-(\bar{b}+\varepsilon)^{2}\right) N>\left((\bar{b}+\varepsilon)^{2}(\underline{b}-\varepsilon)-(\underline{b}-\varepsilon)^{2}\right) M .
\end{gathered}
$$


Choose $L \in((\bar{b}+\varepsilon) M+((\bar{b}+\varepsilon) /(\underline{b}-\varepsilon)) N,(\underline{b}-\varepsilon) N+((\underline{b}-\varepsilon) /(\bar{b}+\varepsilon)) M)$. By (2.33), an integer $N_{0}>n_{0}+d+|\alpha|$ can be chosen such that

$$
\begin{gathered}
\underline{b}-\varepsilon<b_{n}<\bar{b}+\varepsilon, \quad \forall b \geq N_{0} \\
\sum_{t_{1}=N_{0}}^{\infty} \sum_{t_{2}=t_{1}}^{\infty} \cdots \sum_{t_{k}=t_{k-1}}^{\infty} \sum_{t=t_{k}}^{\infty} \frac{q_{t}}{\left|\prod_{i=1}^{k} a_{i t_{i}}\right|} \leq \min \left\{\frac{\underline{b}-\varepsilon}{\bar{b}+\varepsilon} L-(\underline{b}-\varepsilon) M-N, \frac{\underline{b}-\varepsilon}{\bar{b}+\varepsilon} M+(\underline{b}-\varepsilon) N-L\right\} .
\end{gathered}
$$

Define two mappings $T_{1 L}, T_{2 L}: A(M, N) \rightarrow X$ by

$$
\begin{aligned}
& \left(T_{1 L} x\right)_{n}= \begin{cases}\frac{L}{b_{n+d}}-\frac{x_{n+d}}{b_{n+d}}, & n \geq N_{0}, \\
\left(T_{1 L} x\right)_{N_{0}}, & \beta \leq n<N_{0},\end{cases} \\
& \left(T_{2 L} x\right)_{n}= \begin{cases}\frac{(-1)^{k}}{b_{n+d}} \sum_{t_{1}=n}^{\infty} \sum_{t_{2}=t_{1}}^{\infty} \cdots \sum_{t_{k}=t_{k-1}}^{\infty} \sum_{t=t_{k}}^{\infty} \frac{f\left(t, x_{t-r_{1 t}}, x_{t-r_{2 t}}, \ldots, x_{t-r_{s t}}\right)}{\prod_{i=1}^{k} a_{i t_{i}}}, & n \geq N_{0}, \\
\left(T_{2 L} x\right)_{N_{0},} & \beta \leq n<N_{0}\end{cases}
\end{aligned}
$$

for all $x \in A(M, N)$. The rest of the proof is analogous to that in Theorem 2.4. This completes the proof.

Similar to the proof of Theorem 2.7, we have

Theorem 2.8. Assume that there exist constants $M$ and $N$ with $N>((1+\underline{b}) /(1+\bar{b})) M>0$ and sequences $\left\{a_{i n}\right\}_{n \geq n_{0}}(1 \leq i \leq k),\left\{b_{n}\right\}_{n \geq n_{0}},\left\{h_{n}\right\}_{n \geq n_{0}},\left\{q_{n}\right\}_{n \geq n_{0}}$, satisfying (2.2)-(2.4) and

$$
b_{n}<-1 \text {, eventually, }-\infty<\underline{b} \text { and } \bar{b}<-1 \text {. }
$$

Then (1.11) has a bounded nonoscillatory solution in $A(M, N)$.

Remark 2.9. Similar to Remark 2.2, we can also prove that the conditions of Theorems 2.3-2.8 ensure that (1.11) has not only one bounded nonoscillatory solution but also uncountably many bounded nonoscillatory solutions.

Remark 2.10. Theorems 2.1-2.8 extend and improve Theorem 1 of Cheng [6], Theorems 2.1-2.7 of Liu et al. [8], and corresponding theorems in [3, 4, 9-17].

\section{Examples}

In this section, two examples are presented to illustrate the advantage of the above results.

Example 3.1. Consider the following fourth-order nonlinear neutral delay difference equation:

$$
\Delta\left(4^{n} \Delta\left(3^{n} \Delta\left(2^{n} \Delta\left(x_{n}-x_{n-1}\right)\right)\right)\right)=0, \quad n \geq 1 .
$$


Choose $M=1$ and $N=2$. It is easy to verify that the conditions of Theorem 2.1 are satisfied. Therefore Theorem 2.1 ensures that (3.1) has a nonoscillatory solution in $A(1,2)$. However, the results in $[3,4,6,8-17]$ are not applicable for (3.1).

Example 3.2. Consider the following third-order nonlinear neutral delay difference equation:

$$
\Delta\left(\left(2^{n}-n\right) \Delta\left(\left(n^{2}-n+1\right) \Delta\left(x_{n}+\frac{2^{n}-1}{3^{n}} x_{n-4}\right)\right)\right)+\frac{\sin \left(2 x_{n-2}\right)}{n^{2}}-\frac{\cos \left(3 x_{n-3}\right)}{n^{3}}=0, \quad n \geq 5,
$$

where

$$
\begin{array}{cc}
a_{1 n}=n^{2}-n+1, \quad a_{2 n}=2^{n}-n, & b_{n}=\frac{2^{n}-1}{3^{n}} \\
f\left(n, u_{1}, u_{2}\right)=\frac{\sin \left(2 u_{1}\right)}{n^{2}}-\frac{\cos \left(3 u_{2}\right)}{n^{3}}, & h_{n}=q_{n}=\frac{2}{n^{2}} .
\end{array}
$$

Choose $M=1$ and $N=5$. It can be verified that the assumptions of Theorem 2.5 are fulfilled. It follows from Theorem 2.5 that (3.2) has a nonoscillatory solution in $A(1,5)$. However, the results in $[3,4,6,8-17]$ are unapplicable for (3.2).

\section{Acknowledgment}

The authors are grateful to the editor and the referee for their kind help, careful reading and editing, valuable comments and suggestions.

\section{References}

[1] R. P. Agarwal, Difference Equations and Inequalities: Theory, Methods, and Application, vol. 228 of Monographs and Textbooks in Pure and Applied Mathematics, Marcel Dekker, New York, NY, USA, 2nd edition, 2000.

[2] R. P. Agarwal, S. R. Grace, and D. O'Regan, Oscillation Theory for Difference and Functional Differential Equations, Kluwer Academic Publishers, Dordrecht, The Netherlands, 2000.

[3] R. P. Agarwal, E. Thandapani, and P. J. Y. Wong, "Oscillations of higher-order neutral difference equations," Applied Mathematics Letters, vol. 10, no. 1, pp. 71-78, 1997.

[4] R. P. Agarwal and S. R. Grace, "Oscillation of higher-order nonlinear difference equations of neutral type," Applied Mathematics Letters, vol. 12, no. 8, pp. 77-83, 1999.

[5] S. S. Cheng and W. T. Patula, "An existence theorem for a nonlinear difference equation," Nonlinear Analysis: Theory, Methods \& Applications, vol. 20, no. 3, pp. 193-203, 1993.

[6] J. Cheng, "Existence of a nonoscillatory solution of a second-order linear neutral difference equation," Applied Mathematics Letters, vol. 20, no. 8, pp. 892-899, 2007.

[7] I. Györi and G. Ladas, Oscillation Theory of Delay Differential Equations, Oxford Mathematical Monographs, The Clarendon Press Oxford University Press, New York, NY, USA, 1991.

[8] Z. Liu, Y. Xu, and S. M. Kang, "Global solvability for a second order nonlinear neutral delay difference equation," Computers E Mathematics with Applications, vol. 57, no. 4, pp. 587-595, 2009.

[9] Q. Meng and J. Yan, "Bounded oscillation for second-order nonlinear neutral difference equations in critical and non-critical states," Journal of Computational and Applied Mathematics, vol. 211, no. 2, pp. 156-172, 2008.

[10] M. Migda and J. Migda, "Asymptotic properties of solutions of second-order neutral difference equations," Nonlinear Analysis: Theory, Methods \& Applications, vol. 63, no. 5-7, pp. e789-e799, 2005. 
[11] E. Thandapani, M. M. S. Manuel, J. R. Graef, and P. W. Spikes, “Monotone properties of certain classes of solutions of second-order difference equations," Computers \& Mathematics with Applications, vol. 36, no. $10-12$, pp. 291-297, 1998.

[12] F. J. Yang and J. C. Liu, "Positive solution of even-order nonlinear neutral difference equations with variable delay," Journal of Systems Science and Mathematical Sciences, vol. 22, no. 1, pp. 85-89, 2002.

[13] B. G. Zhang and B. Yang, "Oscillation in higher-order nonlinear difference equations," Chinese Annals of Mathematics, vol. 20, no. 1, pp. 71-80, 1999.

[14] Z. Zhang and Q. Li, "Oscillation theorems for second-order advanced functional difference equations," Computers \& Mathematics with Applications, vol. 36, no. 6, pp. 11-18, 1998.

[15] Y. Zhou, "Existence of nonoscillatory solutions of higher-order neutral difference equations with general coefficients," Applied Mathematics Letters, vol. 15, no. 7, pp. 785-791, 2002.

[16] Y. Zhou and Y. Q. Huang, "Existence for nonoscillatory solutions of higher-order nonlinear neutral difference equations," Journal of Mathematical Analysis and Applications, vol. 280, no. 1, pp. 63-76, 2003.

[17] Y. Zhou and B. G. Zhang, "Existence of nonoscillatory solutions of higher-order neutral delay difference equations with variable coefficients," Computers $\mathcal{E}$ Mathematics with Applications, vol. 45, no. 6-9, pp. 991-1000, 2003. 